\title{
Pulmonary Emphysema Induced by Cigarette Smoke Solution and Lipopolysaccharide in Guinea Pigs
}

\author{
Nobuaki Mizutani, ${ }^{*, a}$ Jun-ichi FuchiKami ${ }^{b}$ Maki TaKahashi,${ }^{b}$ Takeshi Nabe, ${ }^{c}$ Shin Yoshino, ${ }^{a}$ and \\ Shigekatsu $\mathrm{KOHNO}^{c}$ \\ ${ }^{a}$ Department of Pharmacology, Kobe Pharmaceutical University; 4-19-1 Motoyamakita, Higashinada, Kobe 658-8558, \\ Japan: ${ }^{b}$ Sugi Institute of Biological Science Co., Ltd.; 10221 Kobuchisawa-cho, Hokuto, Yamanashi 408-0044, Japan: and \\ ${ }^{c}$ Department of Pharmacology, Kyoto Pharmaceutical University; 5 Nakauchi, Misasagi, Yamashina, Kyoto 607-8414, \\ Japan. $\quad$ Received April 24, 2009; accepted June 17, 2009; published online June 18, 2009
}

Exposure of animals to cigarette smoke for longer than 3 months leads to the development of chronic obstructive pulmonary disease (COPD) showing pulmonary emphysema. We attempted to create a COPD model with emphysema that could be established in a shorter period of time. Guinea pigs were intratracheally treated once a day on days $0-3,5-8,10-13$ and $15-18$ with a cigarette smoke solution (CSS), which was prepared by bubbling a stream of smoke into saline. Additionally, lipopolysaccharide (LPS) was administered intratracheally as an exacerbation factor on days 4,9 and 14. By day 19, there was a gradual elevation of specific airway resistance (sRaw). In addition, both residual volume and functional residual capacity were found to be significantly higher on day 19. In the lungs, there was a marked increase in leukocytes, especially neutrophils. Histologically, we observed epithelial hyperplasia and emphysema. On the other hand, daily oral administration of theophylline during the administration of CSS and LPS suppressed the sRaw increase and the epithelial hyperplasia, but not other functional structural changes. In conclusion, we established an experimental COPD model in guinea pigs by using intratracheal instillations of CSS and LPS over a considerably shorter term than has been reported for other models.

Key words cigarette smoke; lipopolysaccharide; emphysema; chronic obstructive pulmonary disease; guinea pig

Chronic obstructive pulmonary disease (COPD) is a major global health problem that is predicted to become the third most common cause of death by $2020 .^{1,2)}$ Nearly $90 \%$ of COPD patients are smokers. ${ }^{3)}$ COPD is a condition characterized by airway obstruction due to narrowing of the small airways that is not fully reversible, airway inflammation, and emphysema-related loss of the elastic recoil of the lung. ${ }^{4-6)}$ Pulmonary emphysema is a major component of COPD and is defined by a destructive change of the pulmonary alveoli and enlargement of the respiratory region of the lung distal to the terminal bronchioles. In addition, residual volume (RV) and functional residual capacity (FRC) are characteristically increased in COPD and are related to the degree of hyperinflation of the lungs, especially when there is predominately emphysema. ${ }^{78)}$ Still, the detailed sequence of events leading to the onset of pulmonary emphysema is not yet fully elucidated.

On the other hand, it has been suggested that the morbidity and mortality of COPD are associated with acute exacerbations. ${ }^{9,10)}$ Some exacerbations of COPD are undoubtedly related to respiratory bacteria, which infect the lower airways and increase overall airway inflammation. ${ }^{11,12)}$ In addition, meta-analyses of antibiotic therapy have indicated an advantage for the use of such interventions, which confirms that bacteria are responsible for the exacerbations in COPD. ${ }^{13,14}$ In studies using guinea pigs and mice, it has been reported that repeated exposure of the animals to lipopolysaccharide (LPS) results in persistent chronic pulmonary inflammation observed in human subjects with COPD. ${ }^{15,16)}$

The use of appropriate experimental COPD animal models is indispensable for the development of new therapeutic drugs and for the analysis of the pathogenesis of the disease. By exposing animals to 3 or more months of cigarette smoke, various experimental COPD models have been devel- oped in guinea pigs, rats and mice. ${ }^{17-24)}$ Although all of these models have emphysematous airspace enlargement, studies on the clinical pathogenesis of COPD would be much more efficient if the emphysematous condition could be experimentally reproduced within a much shorter time period.

In order to shorten the period of development of an experimental COPD model, a more efficient delivery system of the constituents of cigarette smoke into the lung is required. To bypass the problem of using inhaled smoke, which is spontaneously exhaled, we attempted to intratracheally instill a cigarette smoke solution (CSS) over several weeks. Since respiratory infections are assumed to be the main risk factors for exacerbation of COPD, we decided to add LPS to the protocol of the multiple CSS administrations. Through the use of these multiple intratracheal administrations of CSS and LPS, we succeeded in inducing airway obstruction, bronchitis and emphysema over a considerably shorter term as compared to that seen for the other models that are currently in use. On the other hand, theophylline is being commonly used in asthma and COPD patients. It has been reported that longterm theophylline use for COPD patients results in improvement of pulmonary functions, indicating that theophylline has a beneficial effect on exacerbation of COPD. ${ }^{25,26)}$ Therefore, we examined the effect of theophylline on the functional structural changes in our model.

\section{MATERIALS AND METHODS}

Animals Male, 5-week-old, Hartley guinea pigs weighing $300-350 \mathrm{~g}$ were purchased from Japan SLC (Hamamatsu). The animals were housed in a temperature-controlled room at 19 to $25^{\circ} \mathrm{C}$ under a daily 12 -h light/dark cycle. All of the experimental procedures were approved by the Institutional Animal Care and Use Committee of Sugi Institute of 
Biological Science Co., Ltd. (Yamanashi).

Reagents LPS (Escherichia coli, serotype 055:B5) and tert-butoxycarbonyl (Boc)-L-alanine 4-nitrophenyl ester were purchased from Sigma-Aldrich Co. (St. Louis, MO, U.S.A.). Theophylline was purchased from Wako Pure Chemical Industries, Ltd. (Osaka). Hi-Lite cigarettes were purchased from Japan Tobacco Inc. (Tokyo).

By using an air pump to generate suction, CSS was prepared by bubbling a stream of the smoke into saline ( $40 \mathrm{ml} / 40$ cigarettes). It took approximately $3 \mathrm{~min}$ to bubble the smoke from one cigarette into the saline.

Study Design The protocol followed in this study is shown in Fig. 1. CSS $(200 \mu \mathrm{l} /$ animal/time $)$ was intratracheally administered once a day on days $0-3$ without the use of general anesthetics. Subsequently, an LPS solution (500 $\mu \mathrm{g} / \mathrm{ml}, 200 \mu \mathrm{l} /$ animal/time) was intratracheally administered on day 4. These 4 CSS and 1 LPS administrations were regarded as being cycle 1 , with cycles 2 and 3 conducted on days 5-9 and 10-14, respectively. CSS was then administered on days 15-18 (CSS + LPS group). Time-related changes in the specific airway resistance (sRaw) were measured on days $0,4,9,14$ and 19. On days $0,4,9$ and 14, measurements of sRaw were performed prior to the administration of CSS or LPS. On day 19, RV, FRC, number of inflammatory cells in the bronchoalveolar lavage fluid (BALF) and lung histology were investigated. On the other hand, the multiple intratracheal administrations of CSS alone were employed for the purpose of examining the role of LPS, as a risk factor for exacerbation of these symptoms, in this model (CSS group). As a negative control, saline was administered intratracheally once a day on days $0-18$ (control group).

On the other hand, in the preliminary study, when LPS solutions were administered on days 4, 9 and 14, the histopathological changes were not significantly observed on day 19 (J. Fuchikami et al., unpublished data). Therefore, we chose the above three groups in this study.

To evaluate the effect of theophylline on these symptoms in the CSS+LPS group, theophylline $(10 \mathrm{mg} / \mathrm{kg})$ was orally administered once a day $1 \mathrm{~h}$ before the respective intratracheal instillations of CSS or LPS on days $0-18$ (CSS + LPS + Theophylline group). The functional structural changes were investigated on day 19.

Intratracheal Instillation Intratracheal instillation was performed using a conscious guinea pig without surgery, as previously described. ${ }^{27,28)}$ Briefly, after removing food sticking to the inner surface of the oral cavity with cotton wool, the mucosal surface of the oral cavity and larynx was topically anaesthetized by painting of $1 \%$ lidocaine solution absorbed into cotton wool. Then, stainless steel tubing (external diameter: $3.40 \mathrm{~mm}$; internal diameter: $2.84 \mathrm{~mm}$; length: $70 \mathrm{~mm}$ ), the tip of which was bent at an angle of $120^{\circ}$, was inserted into the oral cavity. The tip of the oral cannula was properly attached to the opening of the trachea at the larynx. Teflon tubing $(30 \mathrm{~mm})$ was attached to the other end of the oral cannula, and the inner surface of the Teflon tubing was monitored for moisture indicating expiration by the guinea pig through the oral cannula. After confirming that the guinea pig was breathing through the stainless tubing, CSS or LPS was instilled into the tube with a pipet at a dose of $200 \mu \mathrm{l}$ /animal.

Pulmonary Function Measurements sRaw was measured in conscious guinea pigs by a two-chambered, doubleflow plethysmograph system in accordance with the method of Pennock et al. ${ }^{29)}$ In brief, an animal was placed with its neck extending through the partition of a two-chambered box, and after detection of airflow by sensors attached to both the front and rear chambers, sRaw was then measured using a Data Analyzer Pulmos-I device (M.I.P.S., Osaka)

After the measurement of sRaw on day 19, animals were anesthetized with ketamine hydrochloride $(60 \mathrm{mg} / \mathrm{kg}$; Ketalar $^{\circledR}$, Sankyo Co., Ltd.) and xylazine hydrochloride (8 $\mathrm{mg} / \mathrm{kg}$; Seractal ${ }^{\circledR} 2 \%$, Bayer Co., Ltd.) for cannulation into the trachea. Pulmonary functions, RV and FRC were assessed in anesthetized guinea pigs using a Pulmonary Maneuvers System (Buxco Electronics, Inc., Wilmington, NC, U.S.A.). Software was used to calculate the FRC using Boyle's law, with pressure and thoracic displacement data collected after occluding the airway at the end of expiration. $\mathrm{RV}$ was calculated by subtracting the vital capacity from total lung capacity as determined by the pressure-volume maneuvers.

Leukocytes in BALF Immediately after assessment of pulmonary function, lungs were lavaged via the tracheal tube with saline $(5 \mathrm{ml} \times 2)$. The recovered lavage fluid was centrifuged at $120 \times \boldsymbol{g}$ for $5 \mathrm{~min}$ at $4{ }^{\circ} \mathrm{C}$. The cell pellet was suspended with a defined volume (200-800 $\mu \mathrm{l} /$ sample) of saline. The total leukocyte count in the lavage fluid was determined by staining with Turk's solution. For the differential cell counts, BAL cells were centrifuged on the Settling chamber (Neuro Probe, Cabin John, MD, U.S.A.) at $50 \times$ g for $30 \mathrm{~s}$ at $4{ }^{\circ} \mathrm{C}$, and then the settled leukocytes were stained with Diff-Quik solution (International Reagents, Kobe). Minimums of 500 cells were counted under a microscope and based on their morphological criteria, classified as being macrophages, lymphocytes, neutrophils or eosinophils.

Elastase Activity in BALF Elastase activity in BALF was measured using Boc-L-alanine 4-nitrophenyl ester as a substrate. Samples were diluted ten-fold with saline and then

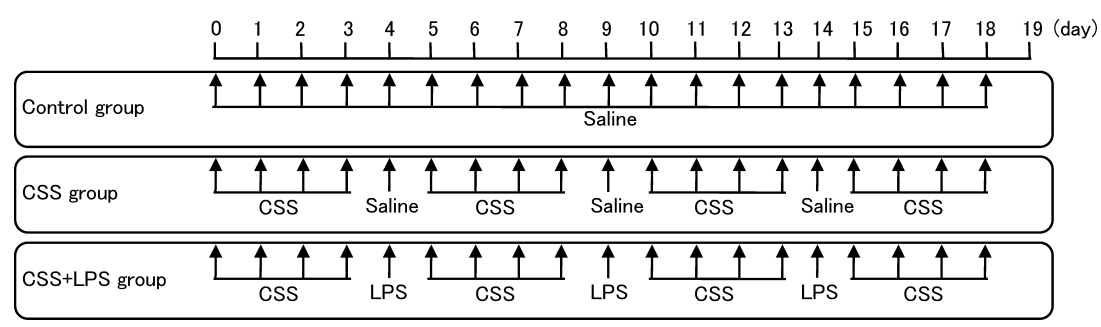

Fig. 1. Schedule for Intratracheal Instillations of CSS and LPS in Guinea Pigs CSS: cigarette smoke solution, LPS: lipopolysaccharide. 
incubated in $0.1 \mathrm{M}$ Tris- $\mathrm{HCl}$ buffer ( $\mathrm{pH} 6.5$ ) containing $0.5 \mathrm{M}$ $\mathrm{NaCl}$ and $1 \mathrm{~mm}$ substrate in a final volume of $1 \mathrm{ml}$ for $4 \mathrm{~h}$ at $37^{\circ} \mathrm{C}$. After incubation, the amount of released $p$-nitroaniline was measured at $405 \mathrm{~nm}$.

Histological Examination of the Lung On day 19, the lung was exposed, and then photographed. Afterwards, the lung was fixed in $10 \%$ buffered formalin and then embedded in paraffin. In order to analyze the morphological changes, $4 \mu \mathrm{m}$ paraffin sections were stained with hematoxylin and eosin (H\&E).

The epithelial hyperplasia in the lung was microscopically assessed using scales from 0 to 3 for severity of change $(0$, No change; 1, Slight change; 2, Moderate change; and 3, Severe change). To assess mean liner intercepts (MLI) as a measure of alveolar size, ten random fields in pulmonary air spaces were evaluated by microscopic projection onto an image analyzer (DP70, Olympus Corporation, Tokyo). The lines were drawn in the images, and intercepts of alveolar walls with these lines were counted. MLI was calculated from the length of the lines multiplied by the number of the lines divided by the sum of all counted intercepts.

Statistical Analyses Data are shown in means \pm S.E. Statistical analyses between the two groups were determined using Student's $t$-test. To compare more than two groups, Dunnett's test was used after doing one-way analysis of variance (ANOVA). A probability value of $p<0.05$ was considered to indicate statistical significance.

\section{RESULTS}

Changes in sRaw, RV and FRC Figure 2 shows the changes in sRaw on days $0,4,9,14$ and 19 during the multiple administrations of CSS with/without LPS. When the administration was repeated, the significant increases in sRaw in both the CSS + LPS and CSS groups were observed on days 14 and 19. In addition, the sRaw elevation in the CSS + LPS group was markedly potentiated compared with the response of the CSS group on day 19.

Figure 3 shows changes in RV and FRC on day 19. The significant elevations of RV and FRC in the CSS + LPS group

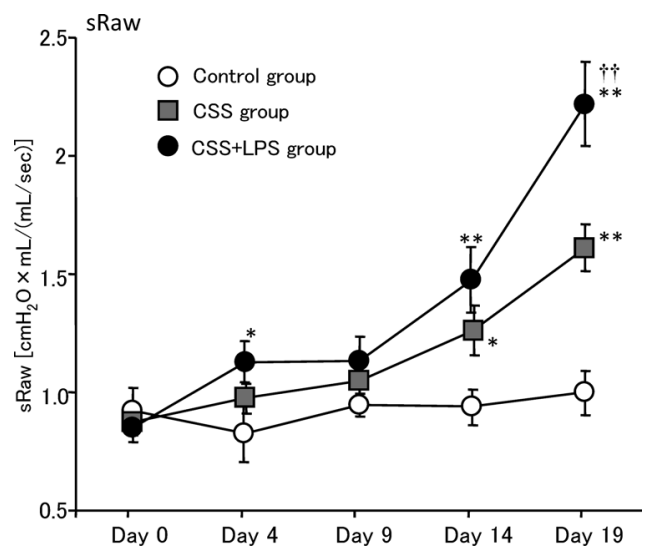

Fig. 2. Time-Course Changes in sRaw during Intratracheal Instillations of CSS and LPS

Each point represents the mean \pm S.E. of 6 to 8 animals. $* p<0.05$ and $* * p<0.01$ : significantly different from the control group. $\dagger^{\dagger} p<0.01$ : significantly different from the CSS group. sRaw: specific airway resistance, CSS: cigarette smoke solution, LPS: lipopolysaccharide. were shown in comparison with the control group. In the CSS group, there was a significant increase in RV, but not FRC. Furthermore, the increases in RV and FRC of the CSS+LPS group were markedly enhanced in comparison with the responses of the CSS group.

On the other hand, in the preliminary study, histological examination showed that the emphysema-related breakdown of the alveolar walls and alveolar expansion in the CSS group was weaker than that in the CSS+LPS group on day 19 , although the degree in the CSS group was significantly higher than that in the control group. However, more repeated instillations of CSS alone (over day 27) gradually aggravated emphysema, which the degree was similar to that in the CSS+LPS group on day 19 (data not shown).

Changes in Leukocyte Numbers in BALF Figure 4 illustrates the changes noted for the total leukocyte, macrophage, lymphocyte, neutrophil and eosinophil counts in the BALF. Although the total number of leukocytes detected in the BALF from the control group was quite small, there were significant increases in the CSS+LPS and CSS groups. The increases in the total number of leukocytes in the CSS+LPS and CSS groups were mainly due to an increase in the number of neutrophils, with the percentage of neutrophils being approximately $50 \%$ in the total leukocyte number. In addition, the number of macrophages, and lymphocytes increased. On the other hand, there was a significant increase in eosinophils in the CSS + LPS group, but not in the CSS group.

Effects of Theophylline Figure 5 illustrates the effect of theophylline on the pulmonary symptoms induced by the instillations of CSS and LPS. Theophylline inhibited the increase in sRaw that was previously noted on day 19 (Fig. 5A). However, theophylline did not inhibit the RV, FRC, and infiltration of the neutrophils (Figs. 5B-D). In addition, theophylline did not suppress the infiltration of the other leukocytes (macrophages, lymphocytes and eosinophils) (data not shown). On the other hand, as compared to the normal group, the level of elastase activity in the BALF of the CSS + LPS group was significantly increased on day 19 . This notable increase was not inhibited by the theophylline treatment (Fig. 5E).

Figure 6 shows the representative appearance and histolog-
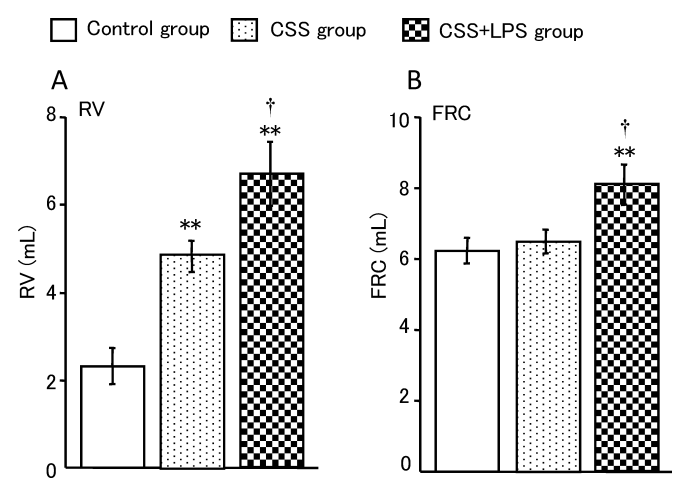

Fig. 3. Changes in RV (A) and FRC (B) Induced by Intratracheal Instillations of CSS and LPS on Day 19

Each column represents the mean \pm S.E. of 6 to 8 animals. $* * p<0.01$ : significantly different from the control group. $\dagger p<0.05$ : significantly different from the CSS group. RV: residual volume, FRC: functional residual capacity, CSS: cigarette smoke solution, LPS: lipopolysaccharide. 

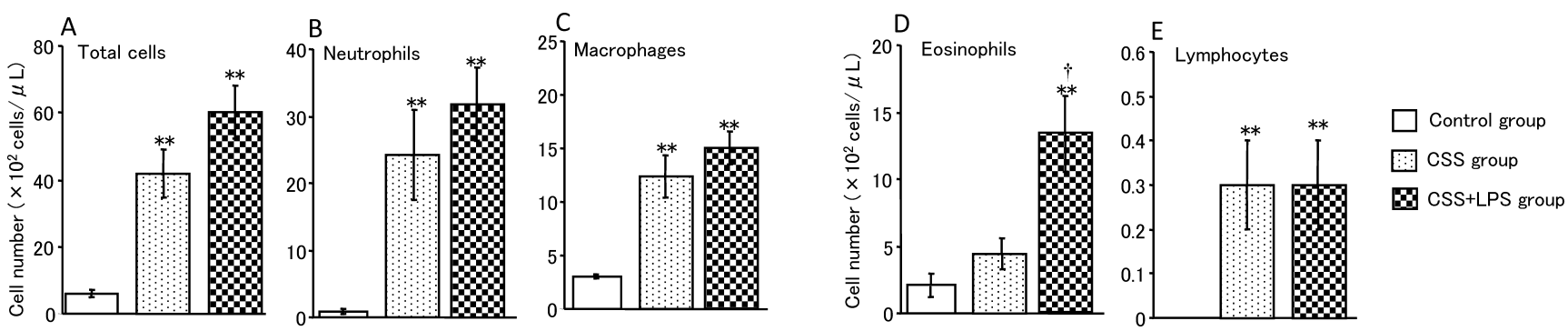

Fig. 4. Changes in Numbers of Total Leukocytes (A), Neutrophils (B), Macrophages (C), Eosinophils (D) and Lymphocytes (E) in BALF Induced by Intratracheal Instillations of CSS and LPS on Day 19

Each column represents the mean \pm S.E. of 6 to 8 animals. $* * p<0.01$ : significantly different from the control group. ${ }^{\prime} p<0.05$ : significantly different from the CSS group. BALF: bronchoalveolar lavage fluid, CSS: cigarette smoke solution, LPS: lipopolysaccharide.
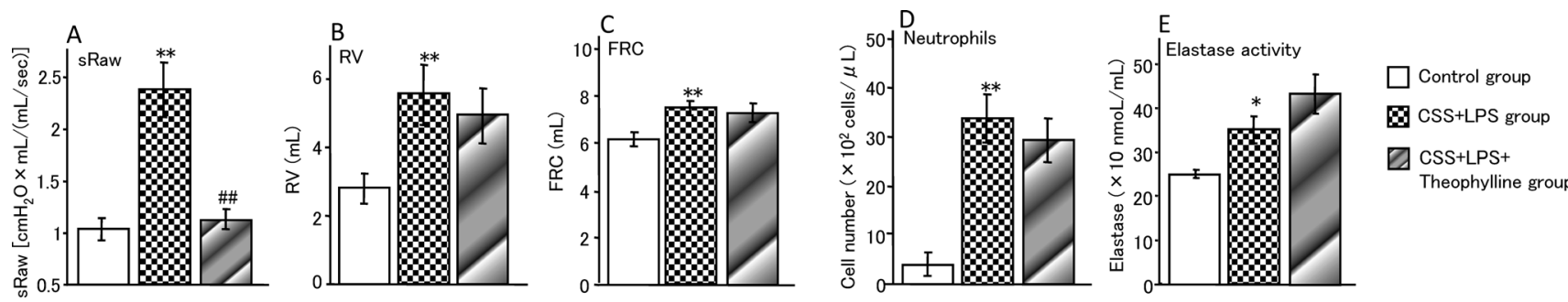

Fig. 5. Effect of Theophylline on Changes in sRaw (A), RV (B), FRC (C), the Number of Neutrophils (D) and Elastase Activity (E) Induced by Intratracheal Instillations of CSS and LPS

Each column represents the mean \pm S.E. of 6 to 8 animals. $* p<0.05, * * p<0.01$ : significantly different from the control group. \# $p<0.01$ : significantly different from the CSS +LPS group. sRaw: specific airway resistance, RV: residual volume, FRC: functional resistance capacity, CSS: cigarette smoke solution, LPS: lipopolysaccharide.
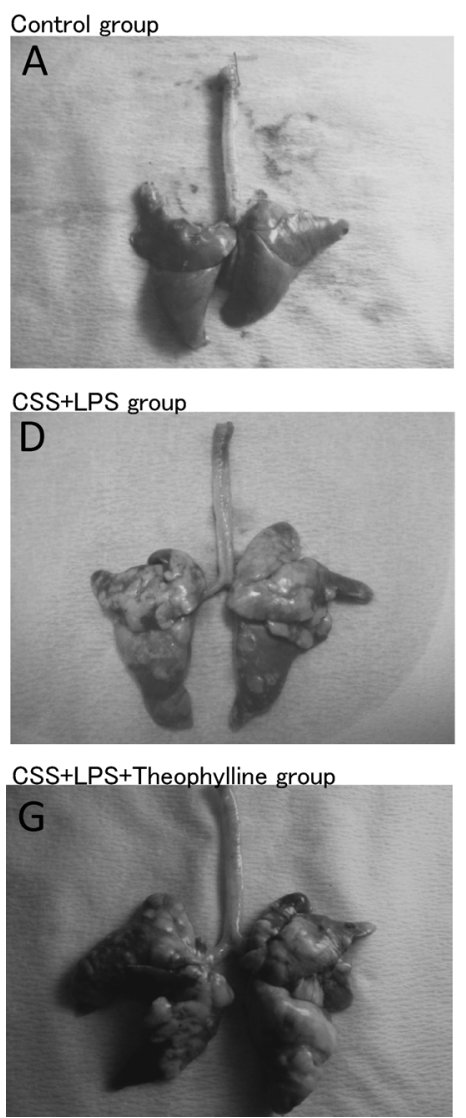
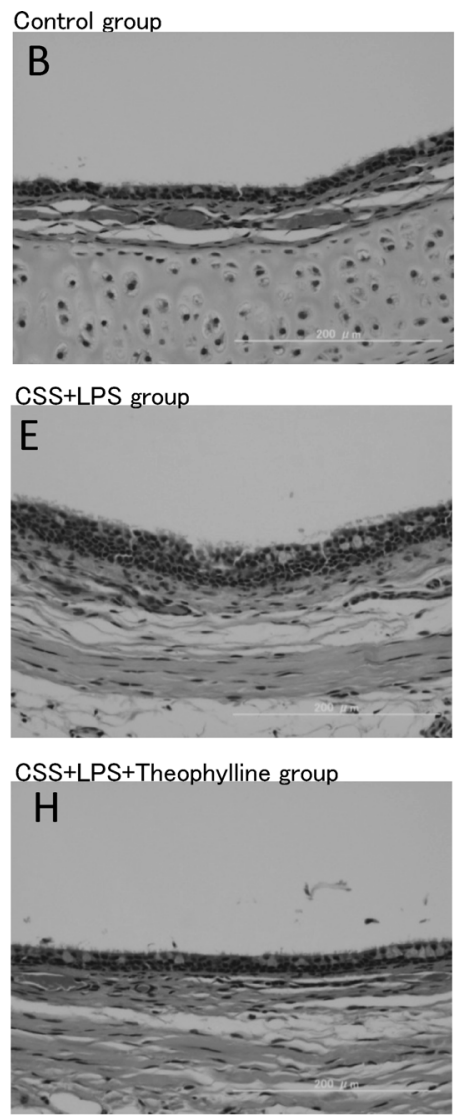
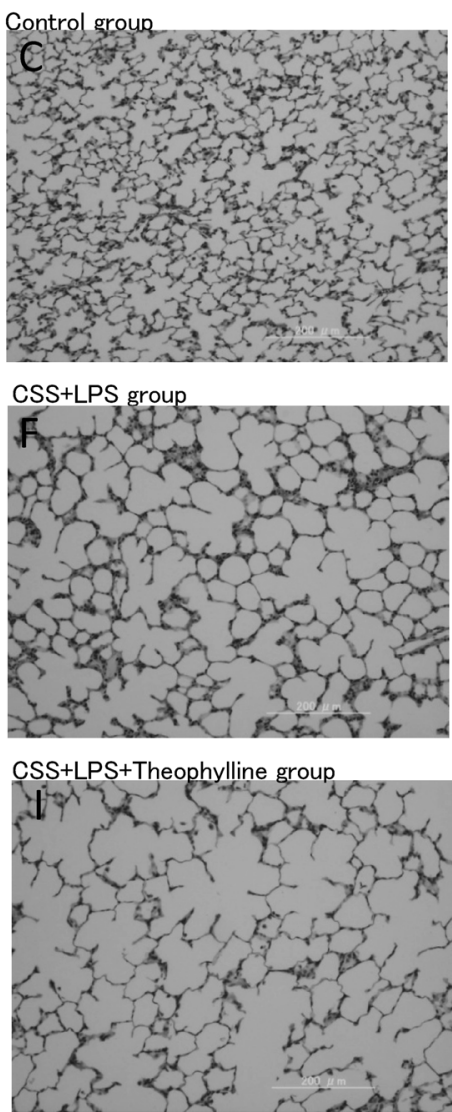

Fig. 6. Effect of Theophylline on the Representative Appearance and Histological Changes in the Lung Induced by Intratracheal Instillations of CSS and LPS

Control group: A, B and C, CSS + LPS group: D, E and F, CSS+LPS+Theophylline group: G, H and I. CSS: cigarette smoke solution, LPS: lipopolysaccharide. 
Table 1. Semiquantitative Analysis of the Effects of Theophylline on Epithelial Hyperplasia Induced by Intratracheal Instillation of CSS and LPS

Mean score

$\begin{array}{ll}\text { Control group } & 0.5 \pm 0.2 \\ \text { CSS + LPS group } & 1.9 \pm 0.1^{* *} \\ \text { CSS + LPS + theophylline group } & 0.8 \pm 0.2^{\# \#}\end{array}$

Data represents the mean \pm S.E. of 6 to 8 animals. $* * p<0.01$ : significantly differen from the control group. $\#^{\prime}<0.01$ : significantly different from the CSS + LPS group CSS: cigarette smoke solution, LPS: lipopolysaccharide.

Table 2. Effect of Theophylline on Changes in Pulmonary Air Space Size Induced by Intratracheal Instillations of CSS and LPS

\begin{tabular}{ll}
\hline \hline & MLI $(\mu \mathrm{m})$ \\
\hline Control group & $45.9 \pm 1.9$ \\
CSS + LPS group & $60.2 \pm 3.7^{* *}$ \\
CSS + LPS + theophylline group & $57.3 \pm 2.5$ \\
\end{tabular}

Data represents the mean \pm S.E. of 6 to 8 animals. $* * p<0.01$ : significantly differen from the control group. CSS: cigarette smoke solution, LPS: lipopolysaccharide, MLI: mean liner intercept.

ical features of the lung on day 19. Pulmonary hyperinflation was observed in the CSS + LPS group (Fig. 6D). On the other hand, for epithelial hyperplasia, we observed increases in the number of epithelial and goblet cells in the bronchi (Fig. 6E, Table 1), and for emphysema, we observed a breakdown of the alveolar walls and alveolar expansion in the lung parenchyma (Fig. 6F). Furthermore, the MLI in the CSS+ LPS group was significantly larger than that in the control group (Table 2). Theophylline inhibited the development of epithelial hyperplasia (Fig. 6H, Table 1). However, theophylline did not inhibit the pulmonary hyperinflation (Fig. $6 \mathrm{G})$, and there was no significant difference in the emphysema-related breakdown of the alveolar walls and alveolar expansion between the CSS+LPS and CSS+LPS+Theophylline groups (Fig. 6I, Table 2).

\section{DISCUSSION}

In the present experiments, we chose the administration of constituents of cigarette smoke intratracheally in order to assure that large fractions of the solution efficiently reached the lower airways and to reduce upper airway response as much as possible. As assessed by changes in sRaw, RV and FRC on day 19, multiple intratracheal administrations of CSS alone produced the increases in sRaw and RV. However, the increase in FRC was not observed. On the other hand, since respiratory infections are assumed to be the main risk factors for exacerbation of COPD, ${ }^{11-14)}$ we added an LPS instillation using the same intratracheal technique during the CSS treatment. As a result, we found that guinea pigs exposed to intratracheal instillations of both CSS and LPS over a 19-d period exhibited COPD-like pathophysiological pulmonary features such as airway obstruction (the increase in sRaw) and pulmonary emphysema (the increases in RV and FRC) that were markedly potentiated compared with responses of multiple administrations of CSS alone.

In contrast to our newly developed model, previous COPD models have used guinea pigs, mice or rats that were fixed to an exposure chamber connected to a smoking machine followed by exposure to a diluted stream of cigarette smoke. ${ }^{17-24)}$ When using the older methodology, it takes more than 3 months to induce emphysema. Therefore, we anticipated that the intratracheal instillation employed in this experiment would deliver the highest possible fraction of an instillation to the lower airways. In addition, we expected that an intratracheal instillation of cigarette smoke solution would result in a prolonged retention of the smoke, which would more effectively elicit lung responses. Furthermore, we believed that exacerbation of the CSS-induced lung injuries could be achieved by additionally instilling LPS using the same technique.

A key finding in the present study is that a combination treatment with CSS and LPS clearly induces emphysema, which is characterized by not only histological changes such as enlargement of lung air space and pulmonary hyperinflation, but also by pulmonary functional changes such as increases in both the RV and FRC. As has been previously suggested, ${ }^{7,8)}$ the increases in RV and FRC that have been noted after exposure to CSS and LPS strongly indicate that this is due to a reduction in the elastic recoil found in the lungs. In addition, instillations of CSS and LPS resulted in a gradual increase in sRaw by day 19, which was indicative of airway obstruction. The degree of increase in sRaw on day 19 was approximately 2 -fold greater than that seen in a model that was developed by exposure to cigarette smoke for 4 weeks. ${ }^{30}$ )

The above-mentioned findings suggest that this experimental COPD model might be a pharmacological tool that can be used to develop therapeutic drugs for treating this disease. Therefore, we examined the effects of theophylline, which is one of therapeutic drugs used in the treatment of COPD. When theophylline was orally administered once a day for $18 \mathrm{~d}$ at a dose of $10 \mathrm{mg} / \mathrm{kg}$, this treatment clearly prevented the elevation of sRaw. Furthermore, theophylline treatment strongly inhibited the development of epithelial hyperplasia. The epithelial hyperplasia that is associated with goblet cell hyperplasia is often closely related to COPD. ${ }^{31}$ ) Kaneko et al. ${ }^{15)}$ reported that goblet cell hyperplasia in LPSinduced lung injury in guinea pigs was inhibited by theophylline. The effectiveness of theophylline in inhibiting both the increase in sRaw and the development of epithelial hyperplasia in our study suggests that the elevation of sRaw may be closely associated with mucus hypersecretion in the airway mucosa. It has been suggested that theophylline's effect may be dependent upon its anti-inflammatory action such as a non-selective phosphodiesterase inhibition and/or histone deacetylase activation, ${ }^{32,33)}$ which can lead to a reduced production of cytokines that contribute to the airway mucus secretion. On the other hand, we found that theophylline did not inhibit the emphysema, which was expressed by increases in RV and FRC, alveolar expansion, and the breakdown of the alveolar walls. The reasons why theophylline did not affect the induction of emphysema are not clear, but this ineffectiveness might explain that theophylline is not an extremely effective treatment in COPD patients.

As of the present time, detailed induction mechanisms for emphysema in this model are unclear. Cigarette smoke-induced emphysema is generally believed to occur as a result of an imbalance between proteases derived from smokeevoked inflammatory cells and antiproteolytic defenses of the lung. ${ }^{34-36)}$ Studies have shown that a proteinase, such as 
elastase, which is released by neutrophils, can induce pathologic changes that resemble human emphysema. ${ }^{18,37)}$ Furthermore, neutrophil sequestration in the pulmonary circulation also leads to the development of emphysema. ${ }^{38)}$ Therefore, these studies confirm that the neutrophils and their proteinases have the potential to produce emphysema. In this model, there were increases in the activity of neutrophils and elastase in the BALF of the CSS+LPS group. Thus, the elastase produced by the neutrophils might be responsible for the induction of the emphysema. Further investigations are needed to determine whether elastase inhibitors can suppress the instillation of CSS and LPS-induced the emphysema.

In conclusion, by employing intratracheal instillations of CSS and LPS, we have created a new experimental COPD model that requires a much shorter developmental time period, as compared to the previously reported COPD models. This model might be useful for elucidating the pathogenesis of COPD, which could possibly lead to the development of new therapeutic drugs.

\section{REFERENCES}

1) Murray C. J., Lopez A. D., Lancet, 349, 1436-1442 (1997).

2) Chung F., Barnes N., Allen M., Angus R., Corris P., Knox A., Miles J., Morice A., O’Reilly J., Richardson M., Respir. Med., 96, 963-975 (2002).

3) Repine J. E., Bast A., Lankhorst I., Am. J. Respir. Crit. Care Med., 156, 341-357 (1997).

4) Cosio M., Ghezzo H., Hogg J. C., Corbin R., Loveland M., Dosman J., Macklem P. T., N. Engl. J. Med., 298, 1277-1281 (1978).

5) Penman R. W., O'Neill R. P., Begley L., Am. Rev. Respir. Dis., 101, 536-544 (1970).

6) Colebatch H. J., Finucane K. E., Smith M. M., J. Appl. Physiol., 34, $143-153$ (1973).

7) Coxson H. O., Rogers R. M., Whittall K. P., D’yachkova Y., Pare P. D., Sciurba F. C., Hogg J. C., Am. J. Respir. Crit. Care Med., 159, 851856 (1999).

8) Newton M. F., O’Donnell D. E., Forkert L., Chest, 121, 1042-1050 (2002).

9) Mannino D. M., Homa D. M., Akinbami L. J., Ford E. S., Redd S. C., Respir. Care, 47, 1184-1199 (2002).

10) Rodriguez-Roisin R., Chest, 117, 398S-401S (2000).

11) Veeramachaneni S. B., Sethi S., COPD, 3, 109-115 (2006).

12) Noweta K., Frankowska M., Grzelewska-Rzymowska I., Pneumonol. Alergol. Pol., 74, 396-402(2006).

13) Anthonisen N. R., Manfreda J., Warren C. P., Hershfield E. S., Harding G. K., Nelson N. A., Ann. Intern. Med., 106, 196-204 (1987).

14) Saint S., Bent S., Vittinghoff E., Grady D., JAMA, 273, 957-960
(1995).

15) Kaneko Y., Takashima K., Suzuki N., Yamana K., Allergol. Int., 56, 445-456 (2007).

16) Vernooy J. H., Dentener M. A., van Suylen R. J., Buurman W. A., Wouters E. F., Am. J. Respir. Cell Mol. Biol., 26, 152-159 (2002).

17) Wright J. L., Churg A., Am. Rev. Respir. Dis., 142, 1422-1428 (1990).

18) Wright J. L., Framer S. G., Churg A., Am. J. Respir. Crit. Care Med., 166, $954-960$ (2002).

19) Kang M-J., Homer R. J., Gallo A., Lee C. G., Crothers K. A., Cho S. J., Rochester C., Cain H., Chupp G., Yoon H. J., Elias J. A., J. Immunol., 178, 1948-1959 (2007).

20) Maeno T., Houghton A. M., Quintero P. A., Grumelli S., Owen C. A., Shapiro S. D., J. Immunol., 178, 8090-8096 (2007).

21) Bracke K. R., D’hulst A. I., Maes T., Moerloose K. B., Demedts I. K., Lebecque S., Joos G. F., Brusselle G. G., J. Immunol., 177, 43504359 (2006).

22) Stevenson C. S., Docx C., Webster R., Battram C., Hynx D., Giddings J., Cooper P. R., Chakravarty P., Rahman I., Marwick J. A., Kirkham P. A., Charman C., Richardson D. L., Nirmala N. R., Whittaker P., Butler K., Am. J. Physiol. Lung Cell Mol. Physiol., 293, L1183-L1193 (2007).

23) Lee J. H., Lee D. S., Kim E. K., Choe K. H., Oh Y. M., Shim T. S., Kim S. E., Lee Y. S., Lee S. D., Am. J. Respir. Crit. Care Med., 172, 987-993 (2005).

24) Sansores R. H., Abboud R. T., Becerril C., Montano M., Ramos C., Vanda B., Selman M. L., Chest, 112, $214-219$ (1997).

25) Murciano D., Auclair M. H., Pariente R., Aubier M., N. Engl. J. Med., 320, 1521-1525 (1989).

26) Guyatt G. H., Townsend M., Pugsley S. O., Keller J. L., Short H. D., Taylor D. W., Newhouse M. T., Am. Rev. Respir. Dis., 135, 1069 1074 (1987).

27) Nabe T., Yamamoto M., Suga M., Kohno S., Eur. J. Pharmacol., 497, 97-104 (2004).

28) Nabe T., Yamauchi K., Shinjo Y., Niwa T., Imoto K., Koda A., Kohno S., Int. Arch. Allergy Immunol., 137, 115-124 (2005).

29) Pennock B. E., Cox C. P., Rogers R. M., Cain W. A., Wells J. H., J. Appl. Physiol., 46, 399-406 (1979).

30) Kubo S., Kobayashi M., Masunaga Y., Ishii H., Hirano Y., Takahashi K., Shimizu Y., Eur. Respir. J., 26, $993-1001$ (2005).

31) Rogers D. F., Eur. Respir. J., 7, 1690-1706 (1994).

32) Barnes P. J., Chest, 129, 151-155 (2006).

33) Nicholson C. D., Shahid M., Pulm. Pharmacol., 7, 1-17 (1994).

34) Shapiro S. D., Clin. Chest Med., 21, 621-632 (2000).

35) Shapiro S. D., Biochem. Soc. Trans., 30, 98-102 (2002).

36) Senior R. M., Anthonisen N. R., Am. J. Respir. Crit. Care Med., 157, S139-S147 (1998).

37) Snider G. L., Stone P. J., Lucey E. C., Breuer R., Calore J. D., Seshadri T., Catanese A., Maschler R., Schnebli H. P., Am. Rev. Respir. Dis., 132, 1155-1161 (1985).

38) Guenter C. A., Coalson J. J., Jacques J., Am. Rev. Respir. Dis., 123, 79-84 (1981). 